\title{
Rapid density fluctuations in the solar wind
}

\author{
P. J. Kellogg ${ }^{1}$ and T. S. Horbury ${ }^{2}$ \\ ${ }^{1}$ University of Minnesota, Minneapolis, MN, 55455, USA \\ ${ }^{2}$ Imperial College, London, UK
}

Received: 10 February 2005 - Revised: 12 November 2005 - Accepted: 15 November 2005 - Published: 23 December 2005

\begin{abstract}
Electron density fluctuations (up to $2.5 \mathrm{~Hz}$ ) in the solar wind have been studied, using the EFW experiment on the Cluster spacecraft, which measures density through measurements of the biased probe potentials relative to the spacecraft. The density fluctuation spectra obtained from the EFW probe potential variations are compared to earlier, OGO 5, measurements of ion density fluctuations and ISEE measurements of electron density fluctuations, and are consistent with them. The electric fields corresponding to the electron density fluctuations are extremely small compared with what would be obtained if the electron fluctuations were not cancelled out by nearly equal ion density fluctuations. This is consistent with the nature of ion acoustic waves. In agreement with ISEE work, the fluctuations are proportional to the ambient density. Correlation with magnetic fluctuations is weak, essentially nonexistent during part of the period studied. This might be expected as magnetic fluctuations are known to be nearly incompressible, but even the correlation with fluctuations in the magnitude of B is very small. However, many structures which apparently are pressure balance structures are found. Pressure balance structures are the nearly perpendicular propagation limit of ion acoustic waves.

As ion acoustic waves are strongly damped in plasmas like the solar wind at least if the plasma is taken as Maxwellian, it has always been a puzzle as to why they are found there. We speculate that these waves are created by mode conversion from magnetic fluctuations, and may represent part of the dissipation process for these.
\end{abstract}

Keywords. Space plasma physics (Electrostatic structures; Turbulence; Waves and instabilities)

\section{Introduction}

Solar Wind density fluctuations in the frequency domain above about $0.01 \mathrm{~Hz}$ have been little studied in situ since the 1980's. They are important for several reasons. (1) Fluctuations in general must replace collisions to account for the validity of MHD, a theory which requires some process to maintain thermal quasi equilibrium, and for violation

Correspondence to: P. J. Kellogg

(kellogg@waves.space.umn.edu) of the conservation of particle magnetic moment and the isotropy of the ion distributions (Kellogg, 2000), (2) Conversion of Langmuir waves to electromagnetic radiation is thought to involve interaction of the Langmuir waves with density ramps, (Kellogg, 1986; Yin and Ashour-Abdalla, 1999; Willes and Cairns, 2003; (3) density fluctuations play an important role in the propagation of radio waves in the solar wind and in near earth space (Lacombe et al., 1997 and references therein), and (4) most of our information about the solar corona inside of $0.3 \mathrm{AU}$ comes from the study of this perturbation of radio signals by density fluctuations (e.g. Woo, 1978, 1995; Coles et al., 1991, 1992; Grall et al., 1996; Kojima et al., 1998).

\section{Observations}

Observations of plasma properties on the time scale near the spin rate are generally quite difficult with a spinning spacecraft. The four Cluster spacecraft are spinning, at $0.25 \mathrm{~Hz}$, but the double probe system of the EFW experiment (Gustafsson et al., 1997), which is used for this study, is designed to be relatively immune to the effects of changing angles to sunlight and to the solar wind. The probes of this system are $8-\mathrm{cm}$ dia. spheres on the end of booms with a tip to tip distance of $88 \mathrm{~m}$.

As the Cluster spacecraft do not go very far beyond the bow shock (to $19 R_{E}$ ), it is not easy to find extended periods when the spacecraft are in the free solar wind, that is, beyond the bow shock and not connected to it by magnetic field. The spacecraft normally only go beyond the bow shock during January-March of each year. A period from 00:03-07:27 UT on 19 February 2002 has been chosen. In Fig. 1 is shown the spacecraft orbit (heavy line) relative to a model bow shock together with a line of magnetic field at a certain time.

In Fig. 2 are shown some data relevant to the plasma surrounding the spacecraft. From bottom to top, the panels are magnitude of the magnetic field in nT from the FGM experiment (Balogh et al., 1997), the electron density taken from the Whisper plasma line, the solar wind speed from the CIS experiment, and the variance of the probe potentials. This last is a measure of the fluctuations of electron density. The Whisper experiment measures the spectrum of Langmuir oscillations in equilibrium with the quasi-thermal 

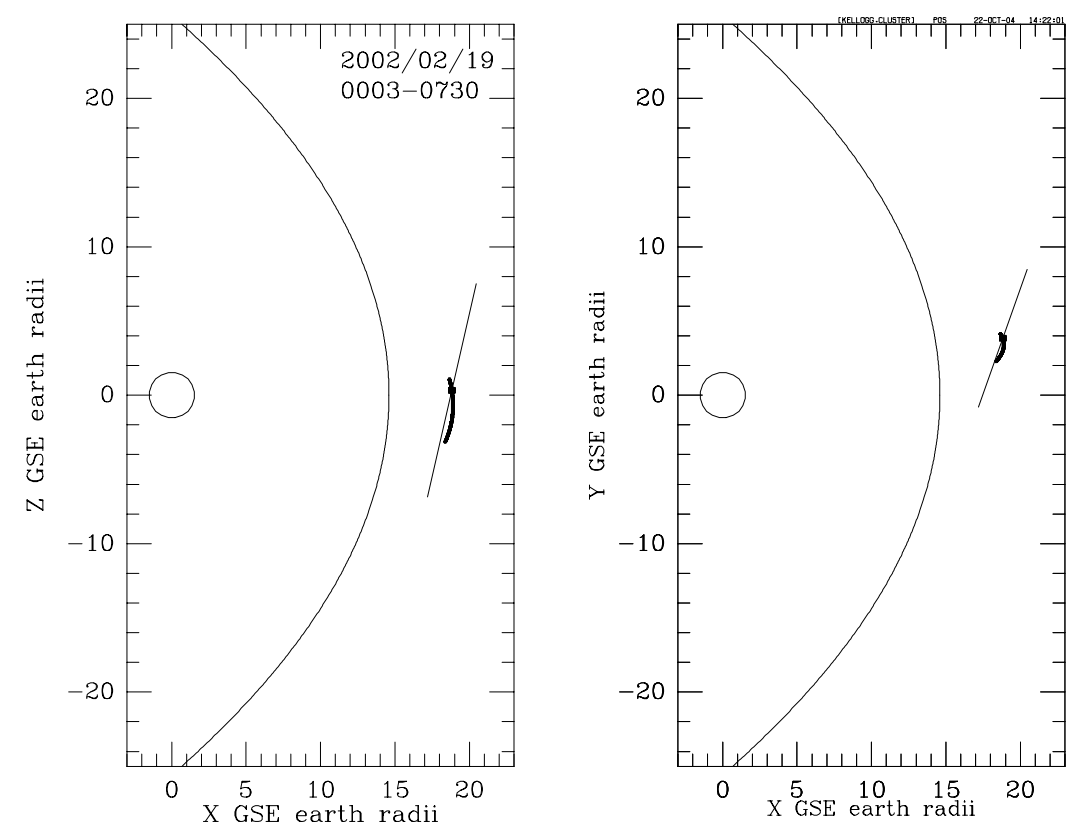

Fig. 1. Spacecraft position during the period chosen for this study, together with a line of magnetic field.

plasma, (Meyer-Vernet, 1979; Kellogg, 1981; Meyer-Vernet and Perche, 1989) which provides a measure of plasma density which is relatively independent of spacecraft perturbations of the plasma, since the Langmuir waves at the lowest frequencies have wavelengths very long compared to the size of the disturbed region. When the spacecraft are in the foreshock region, i.e. when there is a magnetic connection to the bow shock, the amplitude of the plasma line increases enormously, as the plasma is unstable to Langmuir waves in this case. This has been used, together with the occurrence of ion acoustic waves in the Whisper data, to decide whether there is connection to the bow shock and consequent disturbance of the plasma. During the period under study, the solar wind speed was relatively constant, at $335-360 \mathrm{~km} / \mathrm{s}$, so that the dynamic pressure of the solar wind depends mainly on density. Early in the day, the density is about $25-30 \mathrm{~cm}^{-3}$. At $02: 45$, there is a relatively abrupt drop in density to about $10 \mathrm{~cm}^{-3}$. Then at about 03:45 there is a further drop to about $4 \mathrm{~cm}^{-3}$. In response to this decreased dynamic pressure, the bow shock moves out and crosses the Clusters. The satellites remain in the magnetosheath behind the bow shock until about 04:00, reenter the magnetosheath about 04:25 and reemerge into the solar wind at about 05:50. Each of the bow shock crossings is shown by a peak in the variance of the probe potentials. There are bow shock crossings at 03:47, 04:03, 04:18, 05:48, 06:12 and 06:15. During the brief solar wind interval from 04:00 to 04:25, there seems to be some magnetic connection to the bow shock, so that this period is excluded from the study. After 05:50, the bow shock recedes earthward of the satellites, but the density is still lower than earlier, at about $6 \mathrm{~cm}^{-3}$, and remains low until about 07:27. However, there is a magnetic connection to the bow shock until about 06:50, so that only the period from 06:50 to 07:27 has been used. After 07:27, there are some bow shock crossings, and some magnetic connections to the bow shock, and the Clusters are only in the free solar wind for shorter periods for the rest of the day. A heavy line in the lowest panel shows the periods used for this study.

\section{Density vs. probe potential calibration}

In a plasma, an isolated body assumes a potential such that currents to it are balanced. In the solar wind, the main currents to an isolated body are photoelectron emission, which is the largest and constant, and plasma electron pickup. If these are the main contributions, then the body becomes positive to reduce the escape of photoelectrons, by an amount which varies with electron density and temperature. This variation may be reduced nearly to zero by supplying a bias current, in this case to the probes of the double probe sensors, which is constant and nearly balances the photoemission. In that case, the potential of the probe relative to the plasma is constant and small. Pedersen (1995) has shown that geometrical (Langmuir-Blodgett) effects reduce the importance of electron temperature and that the potential of the satellite relative to such a biased probe may be used to measure the electron density.

The EFW experiment supplies such a bias current to the probes, and continuously measures the potential of the probes relative to the main body. We refer to these measurements as "probe potential", but the probe potential relative to the plasma is small and what is of interest is the satellite potential. In the solar wind, the satellites are positive with respect to the plasma, more positive if the density is low. The measured probe potential then is negative and becomes more 

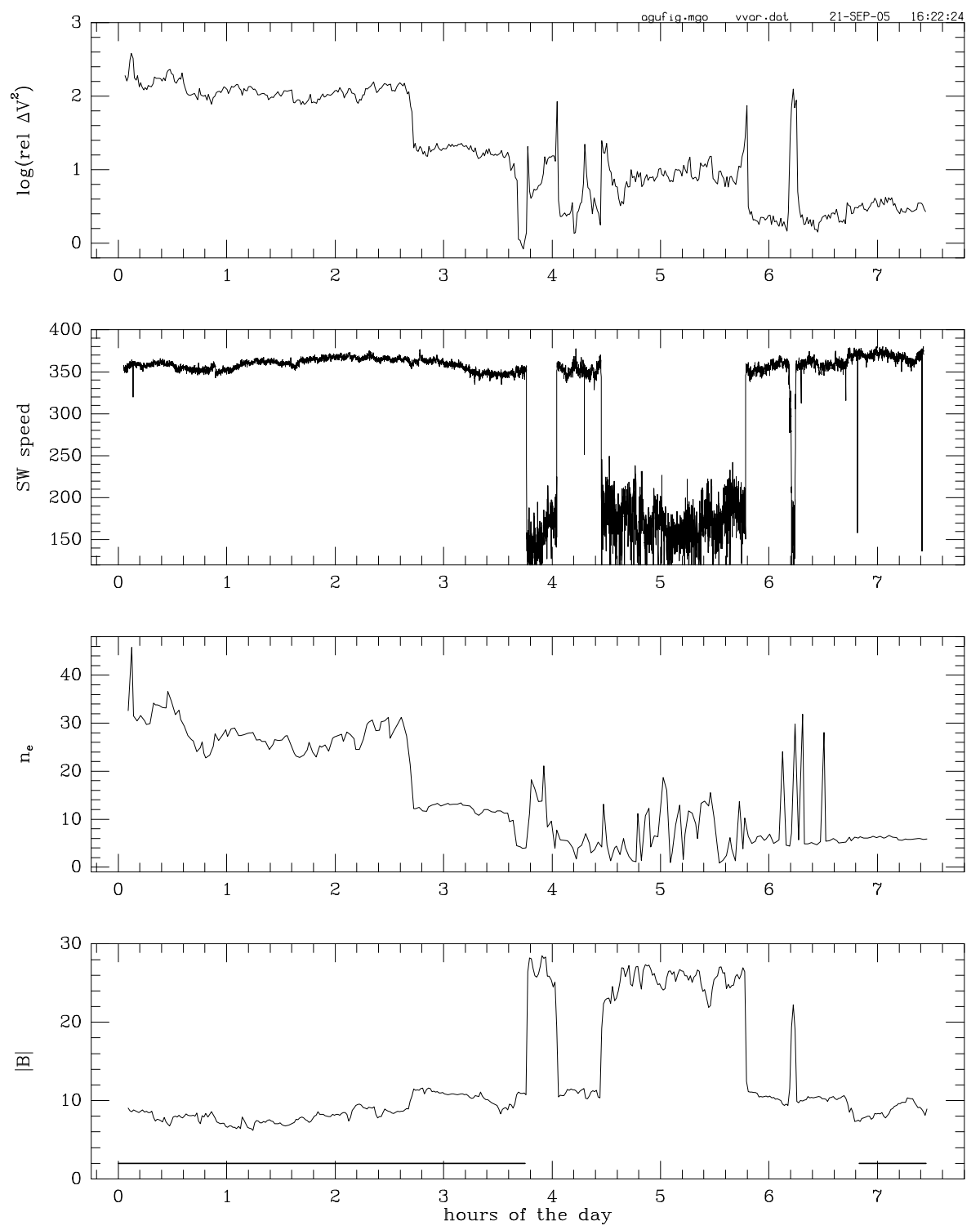

Fig. 2. Some data pertaining to the spacecraft ambiance.

negative as the density becomes smaller, which is convenient intuitively.

In Fig. 3 is shown a set of measurements of probe potential vs. the electron density as obtained from the plasma line as measured by the Whisper experiment (Decreau et al., 1997). It will be seen that the probe potential is a quite good measure of density. In this plot, the periods when Cluster was in the magnetosheath have been excluded. This technique does not work well at all in the magnetosheath, - the points obtained there fall far away from the rest. A. Pedersen (private communication) suggests that secondary emission by energetic electrons in the magnetosheath is responsible. Calibrations similar to these have been carried out by others, (Gustafsson et al., 2001; Pedersen et al., 2001) but it was desired to do a calibration specifically for the conditions of this work. Separate calibrations were made for the various probes. The calibrations differ very slightly.
The curve shows a fit to the observations, a linear combination of three exponentials with different "temperatures" and coefficients, giving six parameters to be established.

\section{Discussion of observations}

It is apparent from Fig. 2 that the variance of the probe potentials is increased when the plasma density is increased. This is shown more clearly in Fig. 4, which shows the density spectra in the regions of Fig. 2, separated according to ambient plasma density. The lowest spectrum (red) is the average of 5 spectra, based on 2048 samples, from the lowest density region, from 06:50 to 07:27 UT, where the average density is about 5-6. The next (orange) is for the period 02:45-03:45 UT, where the average density is about 10-15. The green spectrum is for the period 00:05-02:45 where the 


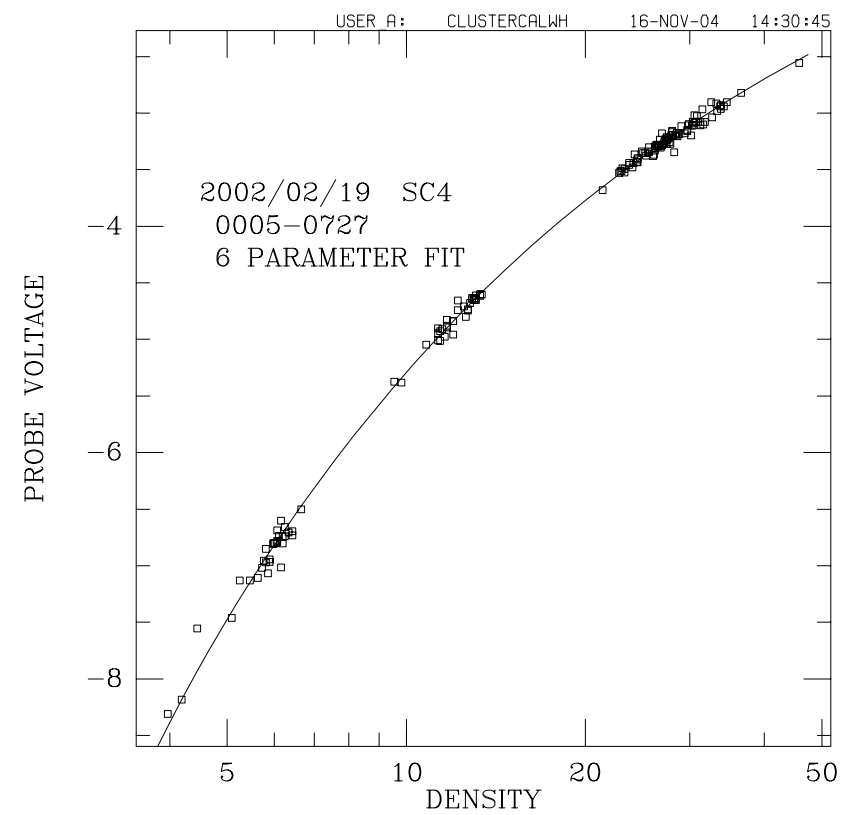

Fig. 3. Calibration of probe voltage vs. density.

average density was $25-40$. The highest spectrum (blue) is the average of 2 large spectra which seemed anomalous, one from the discontinuity at $02: 45$, the other from the beginning of the period, at 00:05 UT, where there is some uncertainty about connection to the bow shock. However, the spectra do not have the form of foreshock spectra and so have been included. In these plots, the spikes are harmonics of the spin frequency, are due to photoelectric effects, and are to be ignored.

Celnikier et al. (1987) were the first to find that the fluctuations are proportional to the density, and this extends their observation to somewhat higher density. In Fig. 5, the results of this technique are compared with earlier measurements of the spectrum of density fluctuations (Unti et al., 1973; Celnikier et al., 1983, 1987). A red line has been drawn at the same frequency and power in each of the plots to facilitate comparison. It will be seen that the present technique yields results which are roughly comparable to the measurements of Unti et al. $(1983,1987)$ who measured ion density fluctuations with a Faraday cup experiment, and with Celnikier et al. $(1983,1987)$, who measured electron density using varying phase shifts in a radio wave propagation experiment. These observers saw the flattening above $0.1 \mathrm{~Hz}$ which is clear in the two spectra at lowest density (red and orange), and Marsch and Tu (1990) have investigated it more completely in Helios data. Here the two lowest densities show similar flattening, but the highest density (green) is well represented by a single power law at $\mathrm{f}^{-1.37}$.

In Fig. 6, plots at a higher time resolution show the approximate proportionality of the "relative power", vs. the square of plasma density averaged over the same $50 \mathrm{~s}$ interval. "Relative power" means that the power has been calculated by summing the squares of the Fourier coefficients of the power

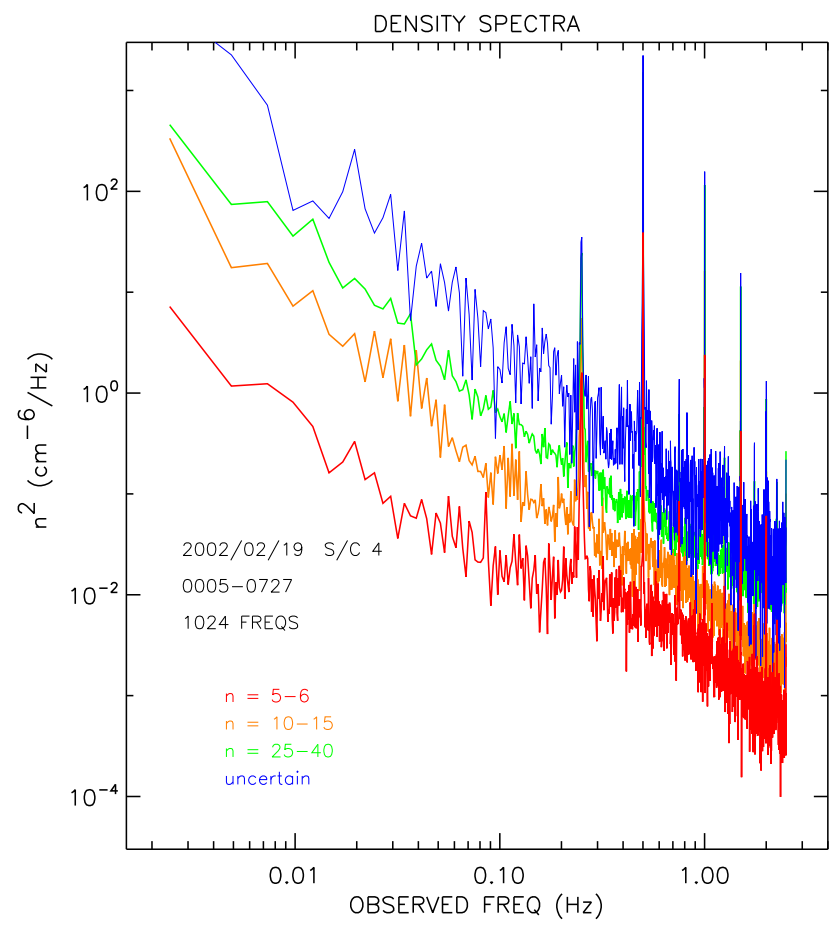

Fig. 4. Density fluctuations spectra in several density regimes.

spectrum weighted by an average spectrum. For the density relative power, the average spectrum is taken to be the power law $\mathrm{f}^{-1.37}$. The result is then essentially the coefficient of a spectral density fitted to the observed spectrum. This is so that all frequencies will be represented in the "relative power", and is used to reduce statistical fluctuation. If one simply calculates the variance of the time series, the descending spectrum means that the low frequency component determines the calculated power, and its statistical fluctuation is so large as to obscure the trends. In calculating relative power, the harmonics of the spin frequency, so prominent in Figs. 4 and 5, have been removed.

The density fluctuations do not seem to follow the magnetic field fluctuations very well. In Fig. 7 are shown time sequence plots of the relative power in the magnetic field fluctuations and in the density fluctuations. Up to about 01:30 UT, there is some tendency to correlation, but then in the period 01:30-02:45, there is anticorrelation, rather. In Fig. 8 is shown a more conventional scatter plot of the correlation, or lack thereof. In this figure, the magnetic field fluctuations are calculated both as the sum of the relative powers in the vector components of $\mathrm{B}$, and the relative power in the magnitude of B. For these "relative powers" of B, the average spectrum has been taken to be a power law at $\mathrm{f}^{-(5 / 3)}$. It would be expected that fluctuations of density would correlate best with fluctuations of the magnitude, i.e. the compressible part. There is little correlation in either case. It will be seen that the relative power in B does not show the same dependence on plasma density as the density power does. But even within the regions of relatively constant ambient density, there is little correlation between the two. Note that 

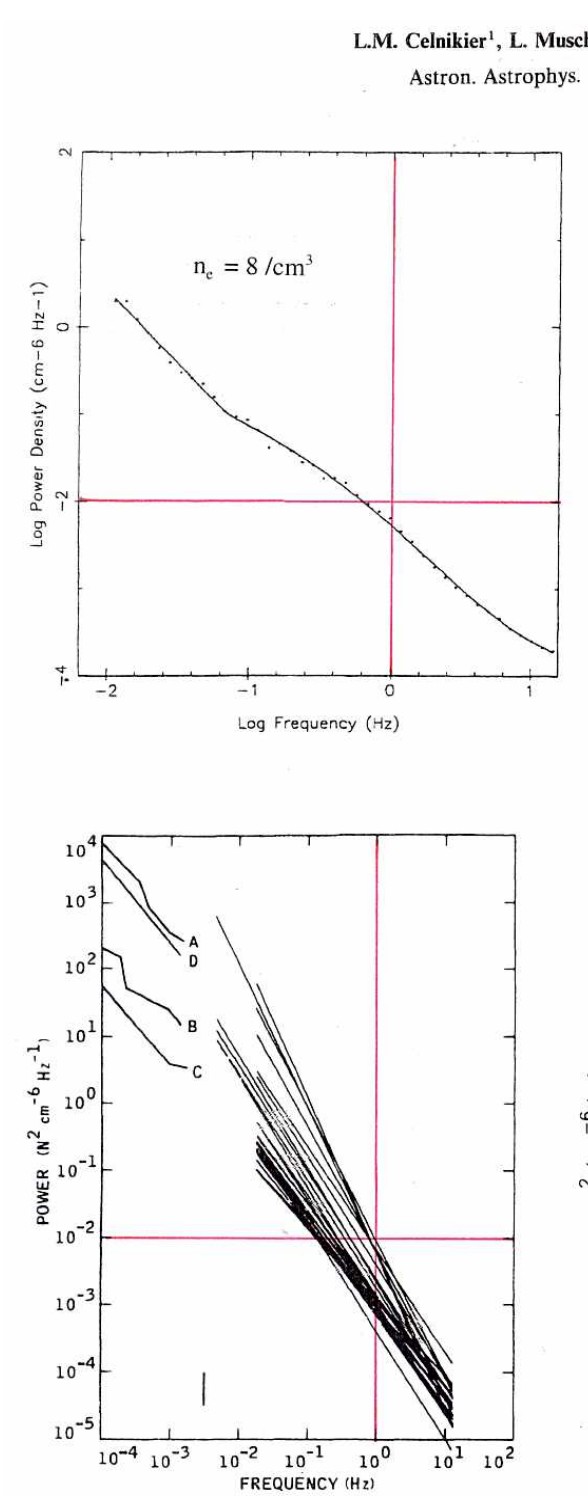

T. W. J. UnTI, M. Neugebauer, AND B. E. Goldstein The Astrophysical Journal, 180: 591-598, 1973

Fig. 5. Comparison with earlier density spectrum measurements.

the period 00:00-01:30 UT corresponds to the highest density, and these points are therefore the points to the right in the figures, since the fluctuations in density are largest then.

Conversely, however, some of the density fluctuations are accompanied by opposite fluctuations in the magnitude of $\mathrm{B}$, which we assume are pressure balance fluctuations or tangential discontinuities. These are very rapid, some changes happening in $0.5 \mathrm{~s}$ or about 5 proton Larmor radii, and since the electron and ion temperatures are not available on the time scale required, this cannot be demonstrated with certainty. But it seems reasonable. Several examples are shown in Fig. 9 In the first 1000 s of 19 February 2002, we identified 30 of these. There were fewer, 7, in the next $1000 \mathrm{~s}$, These are considerably more than the number of discontinuities found in studies of averaged data with a slower cadence,
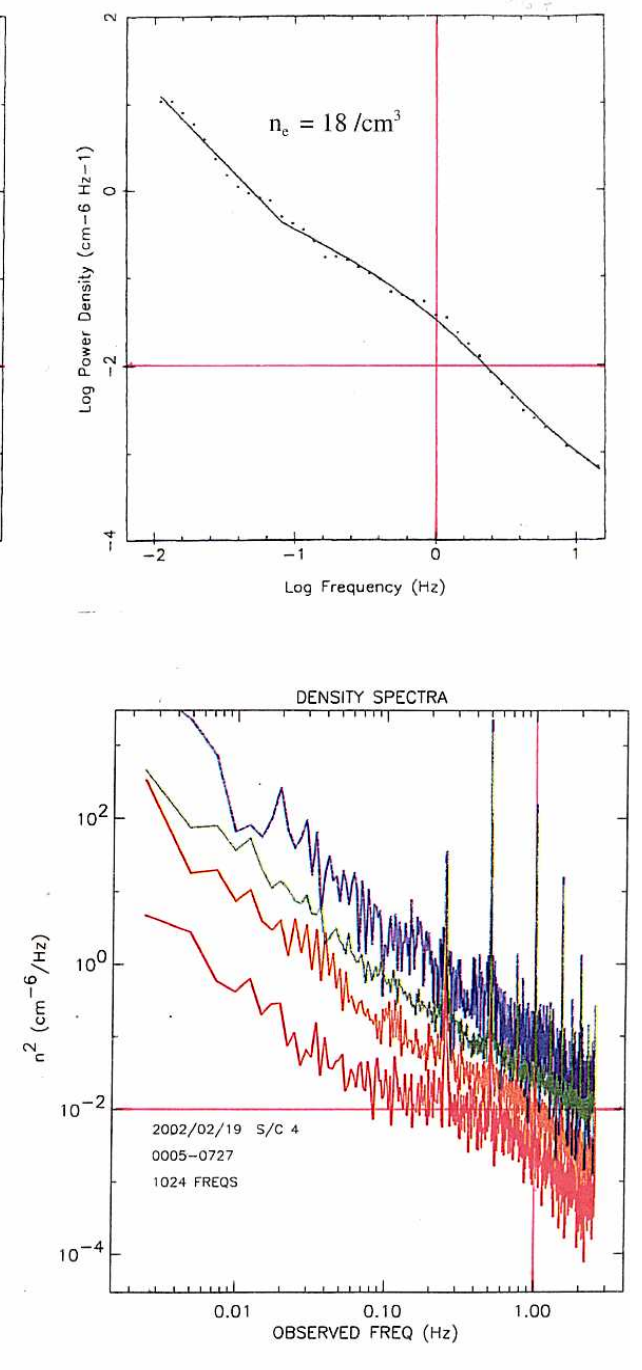

which occur less than once in this time, on average one or two per hour (Smith, 1973; Tsurutani and Smith, 1979; Horbury and Balogh, 2001).

These electron densities must be nearly neutralized by ion densities, a characteristic of electrostatic waves in this low frequency range. If they were not neutralized, then the electric field would be far larger than observed. As an order of magnitude calculation, we have, in Fourier space:

$\mathrm{kE}=-\mathrm{e} \phi \mathrm{n}_{\mathrm{TOT}} / \phi_{0}$.

Assuming convection with the solar wind so that the observed frequency, $f$, is mainly Doppler shift, we have

$\mathrm{k}=2 \phi \mathrm{f} / \mathrm{Vsw} \sim 10^{-5}$ 


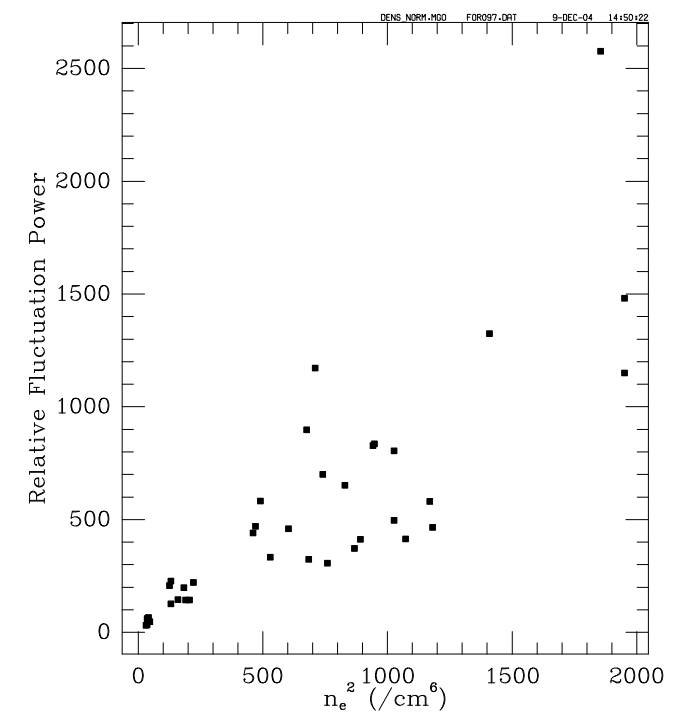

Fig. 6. Dependence of density fluctuations on average density.
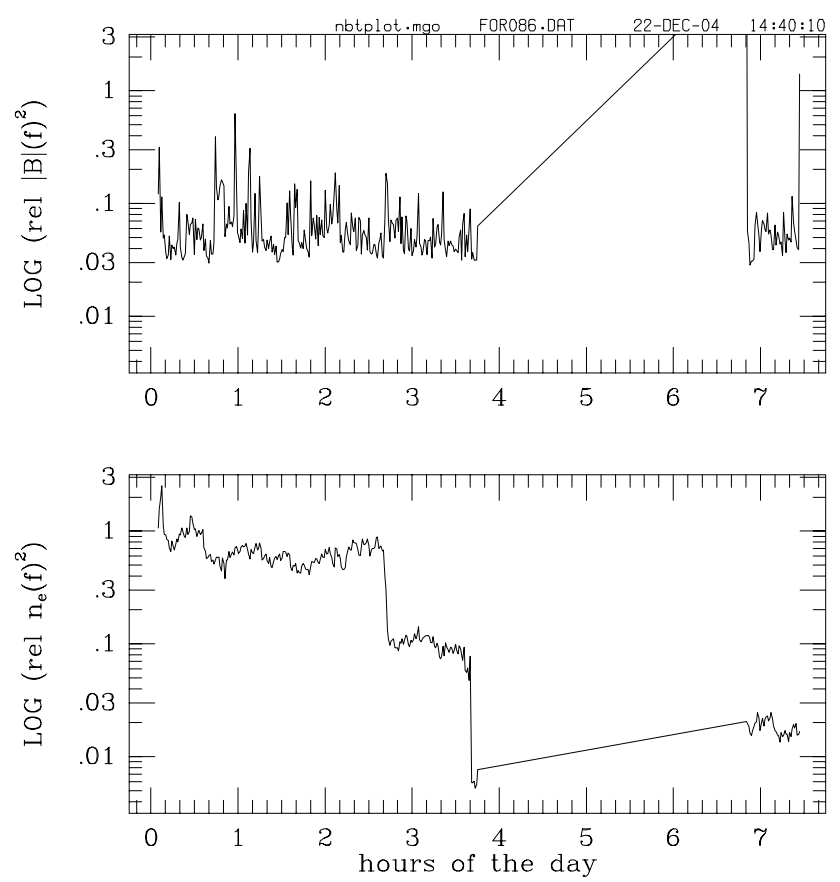

Fig. 7. Time series of relative power in magnetic and density fluctuations.

and taking $\mathrm{dn} \sim 3 \times 10^{-2} / \mathrm{cm}^{6} \mathrm{~Hz}=3 \times 10^{12} / \mathrm{m}^{6} \mathrm{~Hz}$, we would have:

$\mathrm{E}(\mathrm{k})^{2}=\left(\mathrm{e} \delta \mathrm{n} / \mathrm{k} \phi_{0}\right)^{2} \sim 10^{5}(\mathrm{~V} / \mathrm{m})^{2}$.

The electrostatic part of the electric field, which will be the subject of future work, does not exceed about $10^{-9}(\mathrm{~V} / \mathrm{m})^{2} / \mathrm{Hz}$, giving a ratio of greater than $10^{13}$ for the ratio of electron density to charge density divided by e.

This ratio is in rough concordance with expectations for electrostatic waves in which the electrons are isothermal,
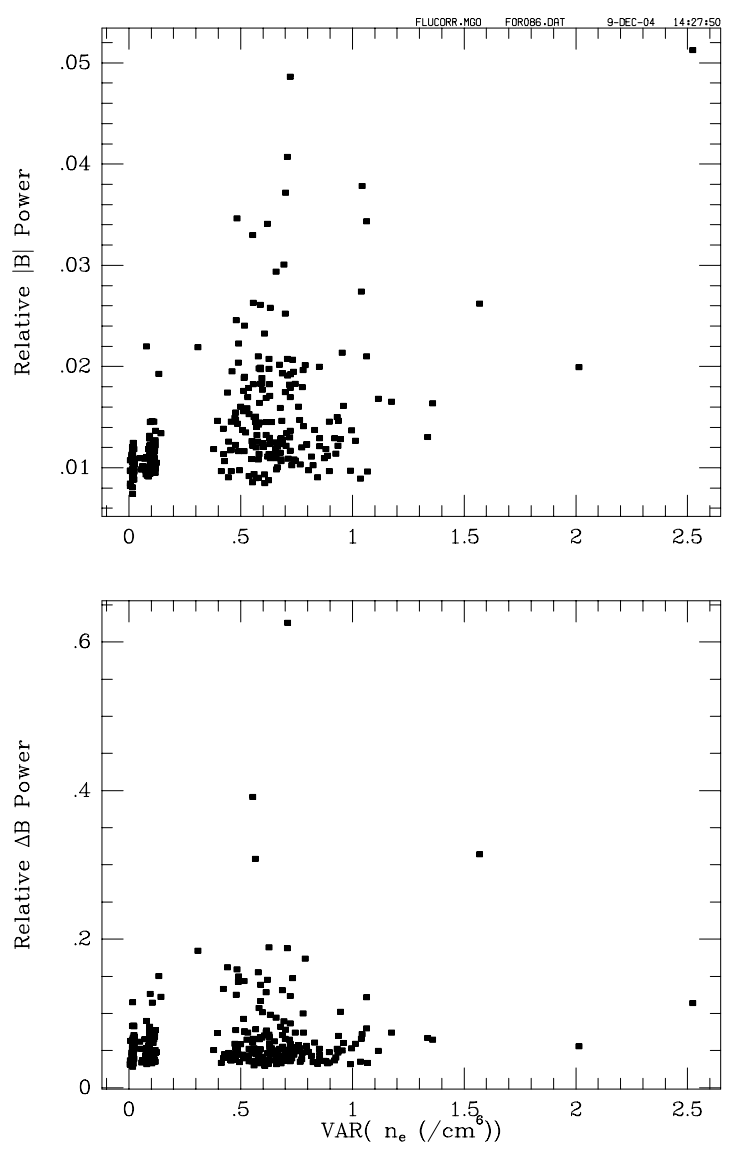

Fig. 8. Scatter plot of the relative power in magnetic and density fluctuations. The lower panel shows the sum of the powers in the three components, the upper shows power in $|\mathrm{B}|$.

such as ion acoustic waves with $\mathrm{k}$ not too close to perpendicular to B:

$\mathrm{n}=\mathrm{n}_{0} \exp (\mathrm{e} \phi / \mathrm{kT})$

$\delta \mathrm{n}_{\mathrm{e}}=\mathrm{n}(\mathrm{e} / \mathrm{kT}) \delta \phi$

which leads to a ratio

$\delta \mathrm{n}_{\mathrm{e}} / \delta \mathrm{n}_{\mathrm{TOT}}=\mathrm{k}^{2} \mathrm{l}_{\mathrm{D}}^{2}$,

where $l_{D}$ is the debye length, here about $10 \mathrm{~m}$, so that $\mathrm{kl}_{\mathrm{D}} \sim 10^{-4}$, and the ratio of the squares of the density fluctuations as above is about $10^{-16}$. A similar very small ratio is also expected for kinetic Alfvén waves at some angles of propagation.

\section{Summary and Conclusions}

We feel that we have verified the probe voltage technique as a measure of electron density fluctuations in the solar wind. There has been, at least for one of us (PJK), always a suspicion that electron temperature might play a greater role than it apparently does. However, the probe voltage curve of 

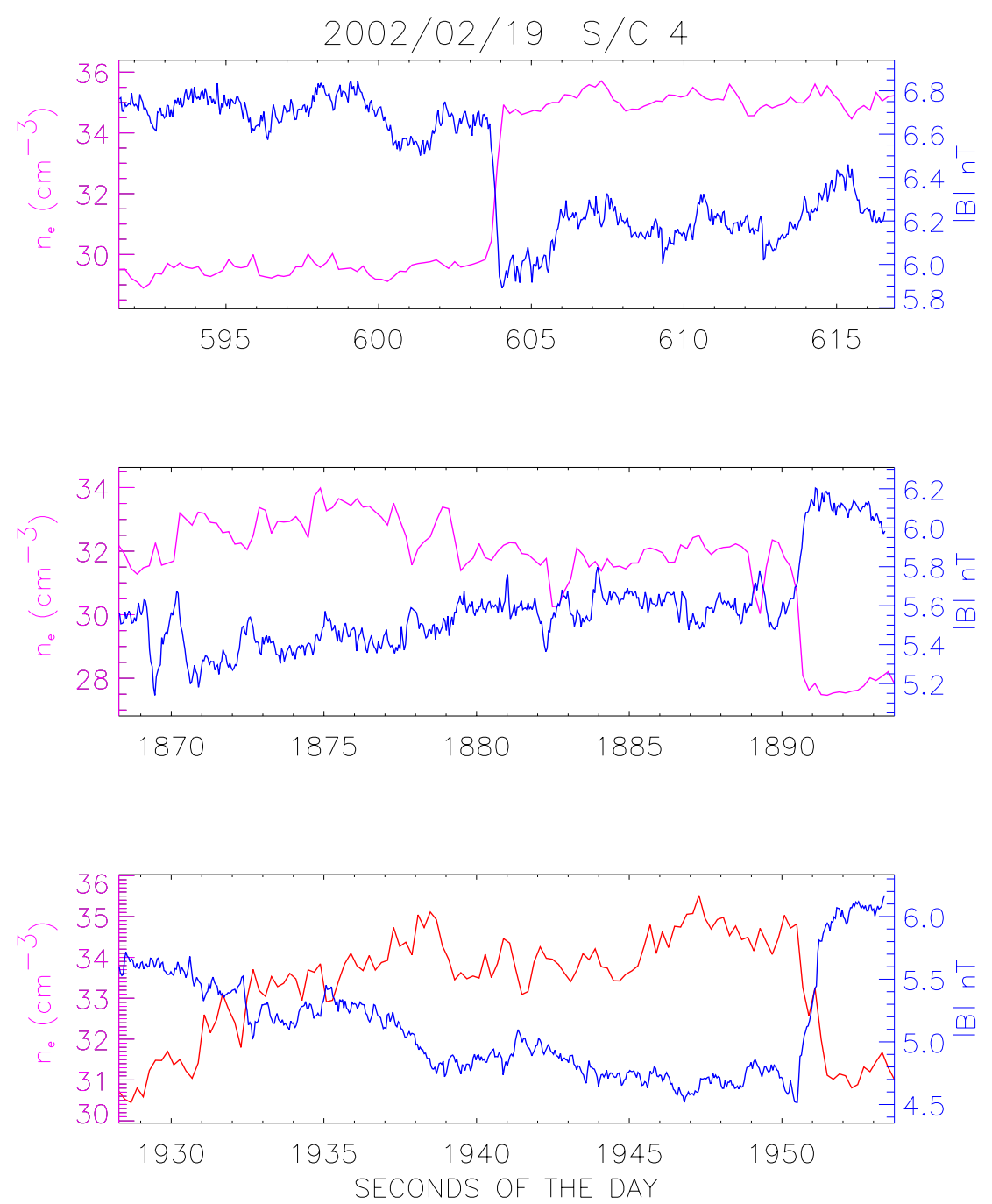

Fig. 9. Probable pressure balance fluctuations.

Fig. 3 shows very little of the scatter that might indicate dependence on electron temperature. The measurements agree with past measurements as well as can be ascertained. Hence we feel that this technique, which offers the possibility of extension to higher frequencies in the future, is validated for the study of fluctuations of density.

A second, physically more important, point is that the density fluctuations represent a plasma mode which is different from the magnetic fluctuations, and which seems not to be included in the frequently used incompressible MHD theory.

Evidence points to density fluctuations as being somewhat like ion acoustic waves, though the evidence is not sufficiently strong to rule out the other mode with strong density fluctuations, the kinetic Alfvén mode at near perpendicular propagation, and certainly not strong enough to rule out a mixture. The electron density fluctuations must be neutralized by ion density fluctuations to high accuracy, as are ion acoustic waves. They are not well correlated with magnetic field fluctuations on the whole, but some fluctuations seem to be pressure balance fluctuations, which do involve magnetic field. This does not contradict the identification with ion acoustic waves, as pressure balance fluctuations are the perpendicular propagation limit of ion acoustic waves. Their power spectrum $\mathrm{f}^{-4 / 3}$ is flatter than that of magnetic fluctuations, which are $\mathrm{f}^{-5 / 3}$ in the lower part of the frequency range studied here, and steeper in the higher part (Leamon et al., 1998, 1999). The power in fluctuations seems to be proportional to the square of the ambient plasma density, in agreement with the observations of Celnikier et al. (1987).

It appears that density fluctuations are relatively independent of magnetic fluctuations. They are even uncorrelated with fluctuations in the magnitude of $\mathrm{B}$, the usual indicator of compressible fluctuations, so that they give another window which can be used to study the turbulent solar wind.

Ion acoustic waves should be heavily damped if the distribution functions of the particles are "normal" Maxwellian distributions. Yet ion acoustic waves do occur frequently in the $\mathrm{kHz}$ range, where they are usually detected through their electric fields. In that range, it is believed that the waves grow on occasionally occurring unstable distributions. The 
$\mathrm{kHz}$ waves are extremely intermittent, however, whereas the waves studied here are less so. It is likely that the two frequency ranges have different causes. As the solar wind is very turbulent, it is probable that there is a lot of mode conversion as well as frequency and wave number conversion taking place, and some of this may result in the conversion of magnetic fluctuations to ion acoustic-like waves which we see here. The inverse process would occur also. A tendency toward equipartition, but involving a balance between mode conversion and wave damping, will result. In this, these ion acoustic-like waves, if they are damped like ion acoustic waves, will be important in the absorption of solar wind fluctuations.

Acknowledgements. The authors would like to thank H. Rème, CESR, France for ion data, Pierrette Decreau LPCE/CNRS, France, for the Whisper data, the U.S. NSSDC and the CSDSweb for data used in several places, S. Bale and F. Mozer of Space Sciences Laboratory, University of California at Berkeley and A. Pedersen, Univerity of Oslo, for invaluable discussions concerning the EFW experiment. This work was supported at the University of Minnesota under NASA contract NAS5-03076.

Topical Editor T. Pulkkinen thanks a referee for her/his help in evaluating this paper.

\section{References}

Balogh, A., Dunlop, M. W., Cowley, S. W. H., Southwood, D. J., Thomlinson, J. G., Glassmeier, K. H., Musmann, G., Lühr, H., Buchert, S., Acuña, M. H., Fairfield, D. H., Slavin, J. A., Riedler, W., Schwingenschuh, K., and Kivelson, M. G.: The Cluster magnetic field investigation, Space Science Reviews, 79(1-2), 65-91, 1997.

Celnikier, L. M., Harvey, C. C., Jegou, R., Kemp, M., and Moricet, P.: A Determination of the Electron Density Fluctuation Spectrum in the Solar Wind, using the ISEE Propagation Experiment, Astron. Astrophys., 126, 293-298, 1983.

Celnikier, L. M.,Muschietti, L., and Goldman, M. V.: Aspects of Interplanetary Plasma Turbulence, Astron. Astroph., 181, 138154, 1987.

Coles, W. A., Esser, R., Lovhaug, U., and Markkanen, J.: Comparison of solar wind velocity measurements with a theoretical acceleration model, J. Geophys. Res., 96(A8), 13 849-13 859, 1991.

Coles, W. A. and Esser, R.: An observational limit to the amplitude of Alfvén waves in the solar wind and comparison with an acceleration model, J. Geophys. Res., 97(A12), 19 139-19148, 1992.

Decreau, P. M. E., Fergeau, P., Krasnosels'kikh, V., Leveque M., Martin, P, Randriamboarison, O., Sene, F. X., Trotignon, J. G., Canu, P., and Mogensen, P. B.: WHISPER, a resonance sounder and wave analyser: performances and perspectives for the Cluster mission, Space Science Reviews, 79, 157-193, 1997.

Grall, R. R., Coles, W. A., Klinglesmith, M. T., Breen, A. R., Williams, P. J. S., Markkanen, J., and Esser, R.: Rapid acceleration of the polar solar wind, Nature, 379(6564), 429-432, 1996.

Gustafsson, G., Bostrom, R., Holback, G., Holmgren, A., Lundgren, A., Stasiewicz, K., Ahlen, L., Mozer, F. S., Pankow, D. B., Harvey, P., Berg, P., Ulrich, R., Pedersen, A., Schmidt, R., Butler, A., Fransen, A. W. C., Klinge, D., Thomsen, M., Falthammar, C.-G., Lindqvist, P.-A., Christenson, S., Holtet, J., Lybekk,
B., Sten, T. A., Tanskanen, P., Lappalainen, K., and Wygant, J.: The electric field and wave experiment for the Cluster mission, Space Science Reviews, 79(1-2), 137-156, 1997.

Gustafsson, G., Andre, M.,Corazzi, T., Eriksson, A. I., Falthammar, C. -G., Grard, R., Holmgren, G., Holtet, J. A., Ivchenko, N., Karlsson, T., Khotyaintsev, Y., Klomov, S., Laakso, H., Lundqvist, P.-A., Lybekk, B., Marklund, G., Mozer, F., Mursula, K., Pedersen, A., Popielawska, B., Savin, S., Stasiewicz, K., Tanskanen P., Vaivads, A., and Wahlund, J.-E.: First results of electric field and density observations by Cluster EFW based on initial months of operation, Ann. Geophys., 19, 1219-1240, 2001 , SRef-ID: 1432-0576/ag/2001-19-1219.

Horbury, T. S. and Balogh, A.: Evolution of magnetic field fluctuations in high speed solar wind streams: Ulysses and Helios observations, J. Geophys. Res, 106(A8), 15 929-15 940, 2001.

Kellogg, P. J.: Calculation and Observation of Thermal Electrostatic Noise in Solar Wind Plasma, Plasma Phys. 23, 735-751, 1981.

Kellogg, P. J.: Observations concerning the generation and propagation of Type III solar bursts, Astron. Astrophysics, 169(1-2, pt.2), 329-335, 1986.

Kellogg P. J.: Fluctuations and ion isotropy in the solar wind, Astrophysical J., 528, 480-485, 2000.

Kojima, M., Tokumaru, M., Watanabe, H., Yokobe, A., Asai, K., Jackson, B. V., and Hick P. L.: Heliospheric tomography using interplanetary scintillation observations, 2. Latitude and heliocentric distance dependence of solar wind structure at 0.1-1 AU, J. Geophys. Res., 103(A2), 1981-1989, 1998.

Lacombe, C., Steinberg J.-L., Harvey C. C., Hubert D., Mangeney, A., and Moncoquet, M.: Density fluctuations measured by ISEE 1-2 in the Earth's magnetosheath and the resultant scattering of radio waves, Ann. Geophys., 15, 387-396, 1997,

SRef-ID: 1432-0576/ag/1997-15-387.

Leamon, R. J., Smith, C. W., Ness, N. F., and Wong, H. K.: Dissipation range dynamics: kinetic Alfvén waves and the importance of beta e/, J. Geophys. Res., 104(A10), 22 331-22 344, 1999.

Leamon, R. J., Smirh, C. W., Ness, N. F., Matthaeus, W. H., and Wong, H. K.,: Observational constraints on the dynamics of the interplanetary magnetic field dissipation range, J. Geophys. Res., 103(A3), 4775-4787, 1998.

Marsch, E. and Tu, C.-Y.: Spectral aand Spatial Evolution of Compressible Turbulence in the Inner Solar Wind, J. Geophys. Res, 95, 11 945-11956, 1990.

Meyer-Vernet, N.:On Natural Noises Detected by Antennas in Plasmas, J. Geophys. Res., 84, 5373-5377, 1979.

Meyer-Vernet, N.and Perche, C.: Tool kit for Antennae and Thermal Noise Near the Plasma Frequency, J. Geophys. Res., 94, 24052415, 1989.

Pedersen, A.:Solar wind and magnetosphere plasma diagnostics by spacecraft electrostatic potential measurements, Ann. Geophys., 13, 118-129, 1995,

\section{SRef-ID: 1432-0576/ag/1995-13-118.}

Pedersen, A., Decreau, P., Escoubet, C.-P., Gustafsson, G., Laakso, H., Lindqvist, P.-A., Lybekk, B., Masson, A., Mozer, F., and Vaivads, A.: Four-point high time resolution information on electron densities by the electric field experiments (EFW) on Cluster, Ann. Geophys., 19, 1483-1489, 2001,

SRef-ID: 1432-0576/ag/2001-19-1483.

Smith, E. J.:Identification of interplanetary tangential and rotational discontinuities, J. Geophys. Res., 78(13), 2054-2063, 1973.

Tsurutani, B. T. and Smith, E. J.: Interplanetary discontinuities: temporal variations and the radial gradient from 1 to $8.5 \mathrm{AU}, \mathrm{J}$. 
Geophys. Res., 84(A6), 2773-2787, 1979.

Unti, T. W. J., Neugebauer, M., and Goldstein, B. E.:Direct Measurements of Solar-Wimd Fluctuations Between 0.0048 and 13.3 Hz, Astrophys. J., 180, 591-598, 1973.

Willes, A. J. and Cairns, I. H.:Banded frequency structure from linear mode conversion in inhomogeneous plasmas, Phys Plasmas, 10(10), 4072-4078, 2003.
Woo, R.:Radial dependence of solar wind properties deduced from Helios 1/2 and Pioneer 10/11 radio scattering observations, Astrophys. J., 219(2, pt.1), 727-739, 1978.

Woo, R.:Solar wind speed structure in the inner corona at 3$12 \mathrm{R}_{\text {sun }}$, Geophys. Res. Lett., 22(11), 1393-1396, 1995.

Yin, L. and Ashour-Abdalla, M.:Mode conversion in a weakly magnetized plasma with a longitudinal density profile, Phys. Plasmas, 6(2), 449-462, 1999. 